\title{
A Search for the Secondary Spectrum of $\epsilon$ Aurigae
}

\author{
P. D. Bennett ${ }^{1,2}$, P. Harmanec ${ }^{3}$, P. Chadima ${ }^{3}$ and S. Yang ${ }^{4}$ \\ ${ }^{1}$ Eureka Scientific, Inc., 2452 Delmer Street, Suite 100, Oakland, CA 94602-3017, USA \\ ${ }^{2}$ Department of Astronomy \& Physics, Saint Mary's University, Halifax, NS, Canada B3H 3C3 \\ email: pbennett@ap.smu.ca \\ ${ }^{3}$ Astronomical Institute of the Charles University, Faculty of Mathematics and Physics, \\ V Holešovičkách 2, CZ-180 00 Praha 8, Czech Republic \\ ${ }^{4}$ Dept. of Physics and Astronomy, University of Victoria, Victoria, BC, Canada V8W 3P6
}

\section{The observations}

The enigmatic long period ( $P=27.1 \mathrm{yr}$ ) eclipsing binary, $\epsilon$ Aurigae, recently emerged from its 2009-2011 eclipse. We have analyzed out-of-eclipse observations (Chadima et al. 2010) obtained over the past 17 years: 306 medium-resolution, high $\mathrm{S} / \mathrm{N}$, spectroscopic observations from $6300-6700 \AA$. Of these, 105 spectra were obtained at the Dominion Astrophysical Observatory (DAO) near Victoria, Canada, from 1994-2010, and 201 spectra were obtained at Ondřejov Observatory (OND), from 2006-2010. Analyzing these data, Chadima et al. (these proceedings) reported on a positive, but ultimately spurious, detection of a secondary spectrum. Their attempts at disentangling the binary spectra were foiled by line profile variations of the F star primary. The $6300-6700 \AA$ spectral region contains several strong stellar lines but space limitations allow us to present only the results for Si II $6347 \AA$. We examine the centroids and higher moments of this prominent F star spectral line for any evidence of a secondary spectrum. Even if secondary contributions are blended with the F star lines, contamination by the secondary star should produce a centroid shift that is anti-correlated with the orbit of the F star primary.

\section{The analysis}

The stellar line profiles observed in $\epsilon$ Aur are quite asymmetric (Figure 1a), and vary on timescales of 50-100d (Chadima et al. 2011), complicating the detection of radial velocity $(\mathrm{RV})$ variability from the companion. Spectra were transformed to the F star reference frame using the Chadima et al. (2010) orbital solution. Observations acquired after the start of spectroscopic eclipse, taken to be JD 2454850 , were discarded because of strong absorption from the companion's disk. A small number $(\sim 10 \%)$ of low S/N observations were omitted. Also, two DAO observations with highly anomalous shifts of tens of $\mathrm{km} \mathrm{s}^{-1}$ were rejected. This left 163 spectra: 76 DAO and 87 OND observations. These spectra were then cross-correlated with DAO or OND reference standards, using a spectral region of weak stellar lines $(6375-6447 \AA)$, to remove intrinsic stellar radial velocity (RV) variations (Figure 1a). The velocity corrections found were typically small $\left(\sim 3 \mathrm{~km} \mathrm{~s}^{-1}\right)$. Then, the first four central moments $\mu_{1}, \cdots, \mu_{4}$, were evaluated, and the line centroid $\mu_{1}$ (Figure $1 \mathrm{~b}$ ), width $\sigma=\mu_{2}^{0.5}$, and skewness $\gamma_{1}=\mu_{3} / \mu_{2}^{1.5}=\mu_{3} / \sigma^{3}$ were derived for each observed profile. We also evaluated mean profiles for each quintile of orbital RV, because any profile anomalies associated with the companion should be negatively correlated with the F star's orbital RV, and thus with the RV quintiles. 

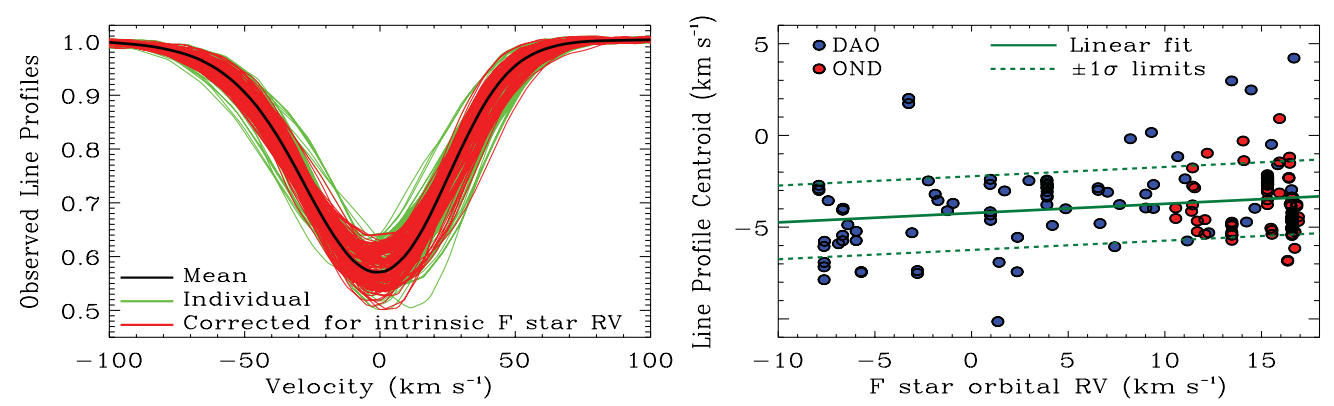

Figure 1. (a) Left: The 163 individual profiles of Si II $6347 \AA$ observed from 1994-2010. Green profiles are in the $\mathrm{F}$ star frame, red are corrected for intrinsic RV variations, the black curve is the mean profile. (b) Right: Centroids of DAO (blue) and OND (red) out-of-eclipse observations.

\section{The results}

The mean line absorption profile of Si II $6347 \AA$ is significantly asymmetric. One possibility is that the intrinsic F star line profiles are symmetric, and these asymmmetries are produced by additional absorption or emission from the companion star. Since any absorption or emission from the companion should be negatively correlated with F star orbital phase, a phase-dependent asymmetry should be present in the observed line profiles. To reduce the observed RV scatter, mean line profiles were computed for observations in each of five equal bins, or quin-

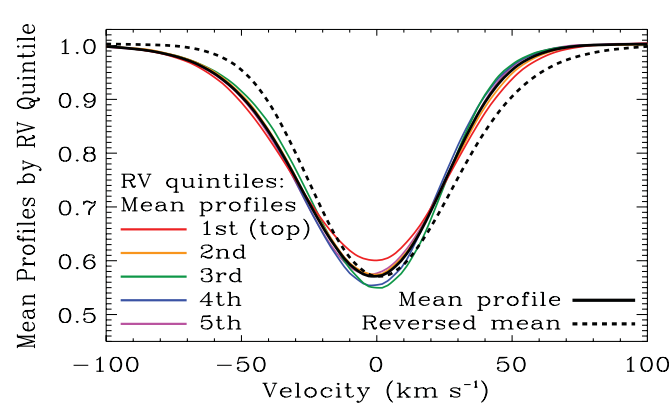

Figure 2. Mean profiles of $\epsilon$ Aur Si II $6347 \AA$, computed over $\mathrm{F}$ star RV quintiles. The overall mean profile is solid black; the mirror-reversed mean profile is the dashed black curve. tiles, of the F star's orbital RV (Fig. 2).

\section{Conclusions}

In the far ultraviolet, $\epsilon$ Aur has an emission line spectrum (Bennett, Ake, \& Harper 2005). However, the quintile centroid shifts of Si II 6347 are too small, and do not show the expected proportional relation to the F star RV, to be consistent with infilling emission. Neither is circumstellar absorption a probable cause, because the line cores are shallowest near the onset of eclipse, when any circumstellar absorption should be large.

We conclude that the line profile variations in the optical spectrum of $\epsilon$ Aur do not arise from contamination by the companion. The asymmetric shape of the F star line profiles is probably intrinsic, as is the variability of these profiles.

\section{Acknowledgements}

PDB and SY acknowledge the support of the NRC (Canada) Herzberg Institute of Astrophysics in the ongoing operation of the DAO $1.2 \mathrm{~m}$ McKellar telescope. PH was supported by grant P209/10/0715 of the Czech Science Foundation.

\section{References}

Bennett, P. D., Ake, T. B., \& Harper, G. M. 2005, BAAS, 37, 495

Chadima, P., Harmanec, P., Yang, S. et al., 2010, IBVS, No. 5937

Chadima, P., Harmanec, P., Bennett, P. D. et al., 2011, A\&3A, 530, A146 